\title{
Using Retrieval Practice to Teach Professionalism to Medical Students: A Proposal
}

\author{
Michael W. Lee ${ }^{1}$
}

Published online: 3 August 2016

(C) International Association of Medical Science Educators 2016

\begin{abstract}
Teaching and assessing professionalism is a priority at medical schools. Approaches taken to teach professionalism include didactic courses, role modeling, clinical experiences, and online modules. However, it remains unclear whether these methods result in long-term retention of professional knowledge and how this, in turn, influences attitudes and behaviors. Recent insight into the effects of repeated testing on social behavior offers an opportunity to enhance the attainment of professionalism. Therefore, harnessing the power of retrieval practice to teach professionalism to medical students over the course of their training may represent an effective means to durably change attitudes and behaviors.
\end{abstract}

Keywords Retrieval practice $\cdot$ Professionalism $\cdot$ Medical students $\cdot$ Ethical behavior

\section{Introduction}

Finding suitable methods to teach professionalism that result in durable changes in student's knowledge, attitudes, and behaviors is a significant challenge in medical education. Although role modeling remains a key way to teach professionalism, it lacks the standardization of other classroom- or electronic-based methods, requires a large degree of time and effort on the part of faculty and clinicians, and the effect of

Michael W. Lee

Lee_Michael@austin.utexas.edu

1 Department of Medical Education, Dell Medical School, University of Texas at Austin, 1501 Red River Street MC: Z0100, Austin, TX 78712, USA role modeling on long-term retention of attitudes and behaviors remains unclear.

Nevertheless, role modeling is a powerful way to influence and guide students in professional behavior and attitude development. As such, it could be paired with other methods of "teaching professionalism" to expand the breadth of topics covered in a learning environment and enhance retention. Therefore, in this commentary, a proposal for developing a method to teach professionalism in a reproducible manner, using professionalism vignettes and retrieval practice to enhance long-term retention, is presented and discussed.

\section{Retrieval Practice, Learning, and Long-Term Retention}

Retrieval practice, also known as the testing effect, is a welldocumented phenomenon in cognitive psychology that has been used in an applied manner in education to increase long-term retention [1-6]. The underlying principle of retrieval practice is that spaced recall or repeated practice in recalling information yields greater long-term memory retention gains compared to repeated study over similar time intervals [7-9]. In other words, taking a test or participating in an activity where one recalls information, rather than studying it over and over again, leads to enhanced long-term retention. The concept of retrieval practice is supported by a large body of research that has shown that the act of retrieving information from memory actually changes memory itself leading to enhanced retention and understanding [10]. Knowledge retention via retrieval also appears to be further enhanced when feedback is provided after the testing/retrieval event [11].

A key component of enhanced long-term retention via retrieval practice is the inclusion of feedback explaining why question answers are right or wrong. To date, most studies 
have focused on retention gains over a week's time using formative or low stakes quizzes on factual knowledge [9]. Recently, Larsen et al. showed retention of knowledge up to 6 months past the initial learning session [2]. Subsequent studies by Larsen et al. demonstrated that retrieval practice is an especially powerful technique for learning when used together with standardized patients [4]. Thus, retrieval practice is a valid means to increase long-term retention of factual information and, indeed, it was recently shown that it can also alter social behaviors [12]. Nevertheless, there is a gap in the literature as to whether or not repeated, interval spaced assessment with feedback would enhance the attainment and long-term retention of professional knowledge in a way that would alter attitudes and behaviors. Although there is evidence in the literature suggesting that assessment with feedback can improve professional behavior, it remains unclear how durable these improvements are and what effect repeated assessment might have on these improvements [13].

\section{What Is Professionalism and Can It Be Taught with Vignettes}

A key provision in testing the benefit of retrieval practice with professionalism is to identify a definition or list of specific professional behaviors to test. Specific definitions for professionalism have remained elusive, and this has resulted in widely differing methods of teaching professionalism in medical schools [14]. Therefore, settling on a "definition" of professionalism is critical in guiding how professionalism is taught and what methods will be employed.

In 2000, Herbert Swick proposed a normative definition of professionalism encompassing nine sets of behaviors professional physicians should exhibit [15]. Because this is a normative definition and, as discussed below, it has previously been assessed with medical students, it is an appropriate starting point for this proposal. The behaviors he articulated state that physicians:

1. Subordinate their own interests to the interests of others

2. Adhere to high ethical and moral standards

3. Respond to societal needs, and their behaviors reflect a social contract with the communities they serve

4. Evince core humanistic values, including honesty, integrity, caring and compassion, altruism and empathy, respect for others, and trustworthiness

5. Reflect upon their actions and decisions

6. Exercise accountability for themselves and for their colleagues

7. Demonstrate a continuing commitment to excellence

8. Exhibit a commitment to scholarship and to advancing their field

9. Deal with high levels of complexity and uncertainty
In a subsequent study, Blue et al. used five of these normative behaviors (self-interest, professional responsibility, managing complexity and uncertainty, professional commitment, and humanism) as a guide and created separate instruments to assess medical students' knowledge and attitudes of professionalism [16]. The instrument they developed to assess professionalism knowledge consisted of 15 professional vignettes where students must chose a single best action or rank the action they would take. They show that in the population of medical students assessed, knowledge of these five behaviors was not uniformly high. In this study, the vignettes they created were used in an assessment capacity; other studies use professional vignettes as a way to teach professionalism to medical students.

Boenink et al. showed that administering professionalism vignettes to pre-clinical medical students enhances knowledge of professional behavior [17]. It is not apparent from this study if feedback was provided or, if so, to what extent. Also, it is important to note that in both of these studies, students were presented with the vignettes once rather than repeatedly over time using different vignettes each time (as is done in retrieval practice). More recently, Greysen et al. developed ten hypothetical "online" professionalism vignettes that were distributed to state medical boards to gage consensus about the likelihood of triggering an investigation [18]. While these authors did not assess the vignettes in a teaching or learning context, they did establish that generation of valid meaningful cases bearing real-world weight is possible. These and other studies suggest, in part, that professionalism can be taught and learned like cognitive, factual-based knowledge making it possible to harness the power of retrieval practice to help students learn to be professional in the pre-clinical years. Therefore, administration of such professionalism-themed vignettes over time that incorporate feedback and lead students to self-reflect together with role modeling could be an economical approach that may ultimately help students and residents exude the nine behaviors articulated by Swick and, in turn, avoid sanctions from state medical boards. However, this remains to be formally assessed. Nevertheless, using online vignette style assessment has some distinct advantages over clinical encounters including time, staffing, and cost.

\section{Implementing Retrieval Practice Using Professionalism Vignettes in a Medical School Curriculum}

A central issue for many medical schools is how to assess and teach professionalism $[14,19]$. However, as mentioned, due to the wide variance in definitions of professionalism, there is a significant degree of heterogeneity among medical schools on how to best accomplish this. Nevertheless, most medical schools have well-established student review boards or panels who review students' cognitive (grades) and non-cognitive 
(professional) performance during their medical school career and render judgments on student sanctions. The long-term consequences of sanctions by these student review boards on physician professionalism and whether they lead to improvements in professionalism following medical school remains unclear. However, professionalism issues that emerge from these reviews could serve as a starting point towards developing vignettes that could be used to instruct students on "best practices". If this were used in tandem with crowdsourcing and social media technology to solicit professionalism "cases" from students or other medical school faculty or administrators, it might be possible to divine the core professionalism issues encountered by medical schools. This would, in turn, aid in the design of professionalism cases or vignette style items that could be used in a formative fashion to teach medical students and residents professionalism. This is important because of mandates from accrediting bodies such as LCME and the community for enhanced accountability for professional training. This, coupled with recent calls for repeated testing in a longitudinal fashion together with feedback and an opportunity for reflection, sets the stage for new studies employing retrieval practice principles [19]. To address this need, I would propose development of a new type of longitudinal formative assessment with vignette style items describing real professional breaches. These formative assessments would be administered in a periodic fashion throughout the 4 years of medical school using different questions/ vignettes that are focused on the same normative behavior (from the nine listed above) for each iteration of the formative assessment. This would help facilitate transfer of learning of previously learned material in a professionalism vignette to new professionalism scenarios relating to the same normative behavior.

A critical component of this strategy is to include feedback for the students on the "correct" answer for the vignette style items that could be released in a controlled time-limited fashion. This has been shown to enhance long-term retention and is reviewed elsewhere [10]. In other words, following completion of the assessment, the students would have a window of opportunity to view feedback for each vignette/item to understand the "correct" way to deal with the professional breach covered. In this way, the formative assessment is not used as a tool to document professional breaches or to "test" a student's professionalism per se but rather as a teaching tool to help students improve their awareness of the types of professional situations encountered in the health sciences and, importantly, how to appropriately respond. It would be interesting to ascertain if administration of these assessments over the course of the training program (i.e., all 4 years of medical school) improves attainment and long-term retention of professional attitudes and behaviors alone or if administering them in tandem with formal teaching or self-learning modules are more effective. Recent published data suggest that retrieval practice assists students in development and maintenance of self- regulated learning [20]. Therefore, coupling self-learning modules with retrieval practice would be predicted to have a synergistic action on the development of these important attributes.

Finally, finding manageable ways to track student performance on professionalism development is a priority. One possibility is that academic assessment offices at medical schools could serve as centralized managers of data from student performance on professionalism vignettes. This performance could be correlated with their performance in clinical settings on rotations. In this way, it might be possible to observe improvements over time. Tracking and observing such changes over longer periods of time (extending into residency and beyond) remains a significant challenge.

\section{Potential Pitfalls and Key Questions to Be Addressed}

Would the application of retrieval practice to professionalism serve as a means to both teach and assess professional attitudes and behavior? The answer to this question will only arise from implementation of such a program followed by rigorous data collection and analysis by multiple medical schools. A key question that needs to be addressed is as follows: What is the optimal spacing interval between professional vignette-based assessments? Although it has been shown that even a single assessment or test can boost longterm retention, repeated testing with long intervals between tests appears to be even more effective [9, 21, 22].

Another issue which needs to be considered is how best to track physicians as they move to residency and, in turn, into clinical practice. At the residency level, implementation of a tracking program or continuation of professionalism testing/ retrieval could prove difficult. However, with the recent release of the AAMC's Core Entrustable Professional Activities for Entering Residency has placed new imperative on tracking and reporting professionalism at the residency level. The emergence of innovative online technology which incorporates retrieval practice in their algorithms has the potential to be an efficient means to deploy professional vignettes to students and address these very questions [23]. A key component of such a system is the ability to adapt to respondents answers and performance. Time will tell if such online platforms will be a successful way to improve professionalism in medical students.

\section{Summary and Conclusion}

In summary, the following schema for harnessing retrieval practice to "teach" professional behavior in a health sciences curriculum is proposed: 
1. Survey faculty and administrators from academic medical centers nationwide for real-world examples of professional breaches and group/categorize according to the six domains of professionalism [24]

2. Assemble multiple formative assessments over time in an adaptable manner using vignettes derived from real-world examples

3. Administer the formative assessments to students in spaced intervals over the course of medical school and residency training with feedback

4. Coordinate with experiential training faculty and standardized patients to ensure application of professional domains covered on the formative assessments

Based on published literature, retrieval practice holds great promise as a means to stimulate and promote long-term retention of professional attitudes and behaviors. By providing students with real life scenarios validated by faculty and administrators at medical schools, students will have the opportunity to set their professional compass to the proper orientation.

In addition, establishing formative feedback assessment tools would lay the foundation for a plethora of studies for determining the long-term outcome of early inculcation of professionalism. This could extend well beyond the "protoprofessional stage" and into residency and post-residency practice. While there are critical parameters that need to be tested, emerging online and mobile technology might hold the key to determining if the retrieval practice would lead to longterm gains in understanding and execution of professional behavior.

\section{References}

1. Dobson JL. Retrieval practice is an efficient method of enhancing the retention of anatomy and physiology information. Adv Physiol Educ. 2013;37(2):184-91.

2. Larsen DP, Butler AC, Roediger 3rd HL. Repeated testing improves long-term retention relative to repeated study: a randomised controlled trial. Med Educ. 2009;43(12):1174-81.

3. Stewart D et al. Pharmacy student self-testing as a predictor of examination performance. Am J Pharm Educ. 2014;78(2):32.

4. Larsen DP et al. The importance of seeing the patient: test-enhanced learning with standardized patients and written tests improves clinical application of knowledge. Adv Health Sci Educ Theory Pract. 2013;18(3):409-25.
5. Dobson JL. Effect of uniform versus expanding retrieval practice on the recall of physiology information. Adv Physiol Educ. 2012;36(1):6-12.

6. Karpicke JD, Blunt JR. Retrieval practice produces more learning than elaborative studying with concept mapping. Science. 2011;331(6018):772-5.

7. Karpicke JD, Roediger 3rd HL. The critical importance of retrieval for learning. Science. 2008;319(5865):966-8.

8. Storm BC, Bjork RA, Storm JC. Optimizing retrieval as a learning event: when and why expanding retrieval practice enhances longterm retention. Mem Cogn. 2010;38(2):244-53.

9. Roediger HL, Karpicke JD. Test-enhanced learning: taking memory tests improves long-term retention. Psychol Sci. 2006;17(3): 249-55.

10. Roediger 3rd HL, Butler AC. The critical role of retrieval practice in long-term retention. Trends Cogn Sci. 2011;15(1):20-7.

11. Butler AC, Roediger 3rd HL. Feedback enhances the positive effects and reduces the negative effects of multiple-choice testing. Mem Cogn. 2008;36(3):604-16.

12. Fernandes M, Saunders J. Does retrieval-induced forgetting affect future social behavior? Acta Psychol. 2013;144(1):1-5.

13. Lynch DC, Surdyk PM, Eiser AR. Assessing professionalism: a review of the literature. Med Teach. 2004;26(4):366-73.

14. Birden $\mathrm{H}$ et al. Teaching professionalism in medical education: a best evidence medical education (BEME) systematic review. BEME guide no. 25. Med Teach. 2013;35(7):e1252-66.

15. Swick HM. Toward a normative definition of medical professionalism. Acad Med. 2000;75(6):612-6.

16. Blue AV et al. Assessment of matriculating medical students' knowledge and attitudes towards professionalism. Med Teach. 2009;31(10):928-32.

17. Boenink AD et al. The effects of teaching medical professionalism by means of vignettes: an exploratory study. Med Teach. 2005;27(5):429-32.

18. Greysen SR et al. Online professionalism investigations by state medical boards: first, do no harm. Ann Intern Med. 2013;158(2): 124-30

19. Goldie J. Assessment of professionalism: a consolidation of current thinking. Med Teach. 2013;35(2):e952-6.

20. Agrawal S, Norman GR, Eva KW. Influences on medical students' self-regulated learning after test completion. Med Educ. 2012;46(3):326-35.

21. Carpenter SK. Cue strength as a moderator of the testing effect: the benefits of elaborative retrieval. J Exp Psychol Learn Mem Cogn. 2009;35(6):1563-9.

22. Carpenter SK, DeLosh EL. Impoverished cue support enhances subsequent retention: support for the elaborative retrieval explanation of the testing effect. Mem Cogn. 2006;34(2):268-76.

23. Dent JA, Harden RM. A practical guide for medical teachers. 4th ed. London: Churchill Livingstone; 2013.

24. Hilton SR, Slotnick HB. Proto-professionalism: how professionalisation occurs across the continuum of medical education. Med Educ. 2005;39(1):58-65. 